\title{
Integration of Transmission Electron Microscopy and Extremophile Virology Research into University and K-12 Education in Maine and Kenya
}

\author{
J. L. Jamison*, K. D. Moulton*, F. E. Riley*, D. L. Ramsdell*, V. M. Serio*, L. J. Rothschild**, \\ F. Mwaura***, and S. M. Duboise* \\ * University of Southern Maine, Department of Applied Medical Sciences, 96 Falmouth Street, 178 \\ Science Bldg., Portland, ME 04103 \\ ** NASA Ames Research Center, Mail Stop 239-20, Moffett Field, CA 94035 \\ *** University of Nairobi, Department of Geography and Environmental Studies, University Way, \\ P.O. Box 30197, GPO100, Nairobi, Kenya
}

Transmission and scanning electron microscopy (TEM and SEM) are enhancing laboratory projectbased learning in molecular biology and virology at the University of Southern Maine (USM) and in the bioscience education outreach activities of the Maine ScienceCorps (MSC) which frequently use microbes and their viruses (bacteriophages) in classroom research projects as previously reported [1]. The MSC at USM is a laboratory intensive NSF-sponsored Graduate STEM Fellows in K-12 Education (GK-12) project that connects graduate students' research to laboratory based projects in Maine's rural high schools. Microbes and their viruses, particularly those isolated from extreme environments, are important in the graduate studies of several MSC Fellows. Research and learning has also been given an international scientific context through parallel investigations of microbial and viral diversity in the acidic mine drainage from Maine's historical iron and copper mines and in soda lakes of the East African Great Rift Valley where extreme alkaline, saline, and thermal conditions are typical. Collaborating with University of Nairobi (UoN) scientists during four trips to Kenya in 2008 and 2009, USM teams including virology and electron microscopy faculty and staff, MSC Fellows, and a participating Maine high school teacher have worked with UoN colleagues to isolate microbes and viruses from Rift Valley soda lakes. During these visits the USM-UoN research team also initiated science education outreach connections in schools and rural education centers in Kenya. Light microscopy resources provided by the research team have been critical in the education outreach that enables students and staff of education centers to image microorganisms important in the ecology of the Rift Valley alkaline lakes. The knowledge of viruses in these environments is to be visually revealed in these educational settings through the TEM images that are produced at USM. TEM images of bacteriophages isolated from the Kenyan soda lakes are shown in Figure 1 and reveal morphotypes that are distinct, but that all are characteristic of the order Caudovirales, the tailed bacteriophages.

The microbial and viral biodiversity of extreme environments in the Great Rift Valley of East Africa and in acidic metal-rich environments such as those in Maine are of significant scientific interest in environmental microbiology and astrobiology with viruses from such environments being particularly under-studied at present. Exemplifying fundamental molecular self organizing properties that are prerequisite for life's origins and propagation, viruses are quintessential molecular models in which self-assembly is genetically encoded into nano-scale biological entities that are the most abundant in Earth's biosphere [2,3]. Through complex interactions with biological hosts and thus ecosystems, viruses are powerful drivers of genetic transfer and evolutionary change, of niche adaptation within diverse habitats, and of nutrient cycling in food webs [4]. As study of virus model systems informed development of molecular and cellular biology [5], the same may be true for 
understanding evolutionary, genetic, and ecological foundations of life in Earth's extreme environments in addressing fundamental questions of astrobiology [6]. Building upon successful efforts in cultivation of the viruses shown in Figure 1 from the extreme environments of Kenya's soda lakes (which at pH 11 and above are among the most alkaline on Earth), the USM virology, microbiology and electron microscopy research team has begun to investigate the molecular genetics and the physical and biochemical parameters internal and external to host cells that determine the structural integrity and functionality of viruses in these extreme environments. Parallel research and education efforts in Maine and in Kenya discussed here are expanding the integrated research and education efforts (some reported previously $[1,7,8,9]$ ) of the USM Virology and TEM laboratory.

References

[1] S.M. Duboise et al., The Science Teacher April-May (2009) 32.

[2] H. Gaussier et al., J. Mol. Biol. 357 (2006) 1154.

[3] C.A. Suttle, Nature 437 (2005) 356.

[4] F. Rohwer and R.V. Thurber, Nature 459 (2009) 207.

[5] J. Cairns et al., Phage and the Origins of Molecular Biology, Cold Spring Harbor Press, 1992.

[6] D.J. Des Marais et al., Astrobiology 8 (2008) 715.

[7] K.D. Moulton et al., Microscopy and Microanalysis. 15, (Suppl. 2), (2009) 956.

[8] V.M. Serio et al. Microscopy and Microanalysis. 15, (Suppl. 2), (2009) 1148.

[9] V.M. Serio et al. Microscopy Today 17(6) 2009) 40.

[10] The Maine ScienceCorps GK-12 Fellowship Program is supported by National Science Foundation grant DGE-0440560, international supplement (DGE-0749059), and USM. TEM capabilities at USM were established with combined NSF (CNS-0521262) and USM support. The Maine Space Grant Consortium and NASA EPSCoR RID Grants EP-07-03 and EP-08-02 have funded Astrobiology research at USM. Teacher professional development programs and SEM resources are funded by grants (R25RR024280 and 3R25RR024280-0 2S1) from the NCRR, a component of the NIH. Content is the responsibility of the authors and does not necessarily represent the views of the funding agencies.

A

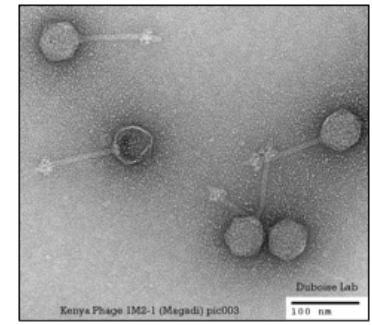

$\mathrm{D}$

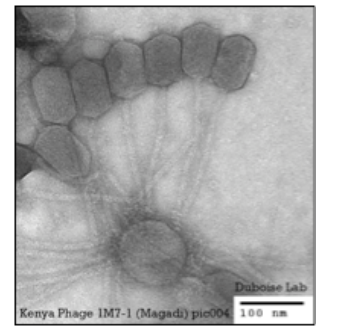

B

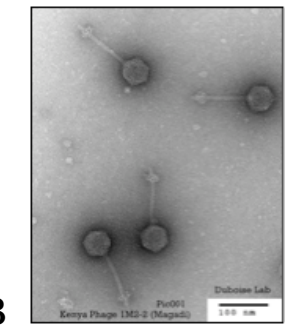

C

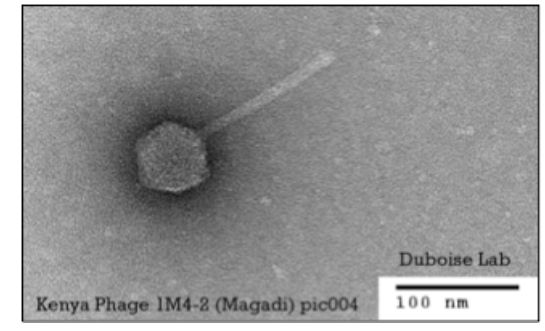

$\mathrm{E}$

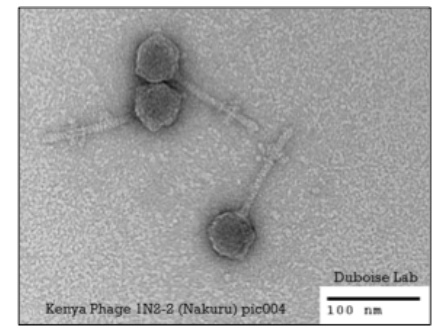

Figure 1: 1\% UA stained TEM images of four bacteriophage isolates that infect microbes from

Kenya's Lake Magadi, panels A-D, while one virus from Kenya's Lake Nakuru is shown in panel E. All bacteriophages shown have morphotypes consistent with the order Caudovirales. 